\title{
ISPRS BENCHMARK FOR MULTI-PLATFORM PHOTOGRAMMETRY
}

\author{
F. Nex ${ }^{\mathrm{a}}$, M. Gerke ${ }^{\mathrm{b}}$, F. Remondino ${ }^{\mathrm{a}}$, H.-J. Przybilla ${ }^{\mathrm{c}}$, M. Bäumker $^{\mathrm{c}}$, A. Zurhorst ${ }^{\mathrm{d}}$ \\ a 3D Optical Metrology (3DOM) unit, Bruno Kessler Foundation (FBK), Italy \\ franex@fbk.eu,remodino@fbk.eu \\ ${ }^{\mathrm{b}}$ University of Twente, Faculty of Geo-Information Science and Earth Observation (ITC), \\ Department of Earth Observation Science, The Netherlands m.gerke@utwente.nl \\ ${ }^{\mathrm{c}}$ Hochschule Bochum, Faculty of Geodesy, Bochum, Germany, heinz-juergen.Przybilla@hs-bochum.de \\ d Aerometrics GmbH, Werne, Germany, azurhorst@vermessung-zurhorst.de
}

Commission III, WG III/1

KEY WORDS: Photogrammetry, Matching, Orientation, Data, Integration, Multisensor, Accuracy, Estimation

\begin{abstract}
:
Airborne high resolution oblique imagery systems and RPAS/UAVs are very promising technologies that will keep on influencing the development of geomatics in the future years closing the gap between terrestrial and classical aerial acquisitions. These two platforms are also a promising solution for National Mapping and Cartographic Agencies (NMCA) as they allow deriving complementary mapping information. Although the interest for the registration and integration of aerial and terrestrial data is constantly increasing, only limited work has been truly performed on this topic. Several investigations still need to be undertaken concerning algorithms ability for automatic co-registration, accurate point cloud generation and feature extraction from multiplatform image data. One of the biggest obstacles is the non-availability of reliable and free datasets to test and compare new algorithms and procedures.

The Scientific Initiative "ISPRS benchmark for multi-platform photogrammetry", run in collaboration with EuroSDR, aims at collecting and sharing state-of-the-art multi-sensor data (oblique airborne, UAV-based and terrestrial images) over an urban area. These datasets are used to assess different algorithms and methodologies for image orientation and dense matching. As ground truth, Terrestrial Laser Scanning (TLS), Aerial Laser Scanning (ALS) as well as topographic networks and GNSS points were acquired to compare 3D coordinates on check points (CPs) and evaluate cross sections and residuals on generated point cloud surfaces. In this paper, the acquired data, the pre-processing steps, the evaluation procedures as well as some preliminary results achieved with commercial software will be presented.
\end{abstract}

\section{INTRODUCTION}

\subsection{Motivations}

In the last years we are witnessing a closer integration of multiplatform and multi-perspective aerial and close-range photogrammetry. This is due to the great developments of imaging sensors and software solutions and to the awareness of the potentials of data integration methods. The introduction of UAV platforms and multi-head oblique image systems mounted on manned airplanes allow the collection of more detailed and complete information compared to traditional image acquisition flights. These new types of data are closing the gap between terrestrial and traditional aerial imaging, becoming the starting point for new research and the development of new applications. The integration will be helpful for a broad range of applications, like city modelling, where a seamless geometric representation at different resolutions is desired. Another example is cultural heritage documentation, where for instance a selection of buildings is modelled in very high detail from terrestrial and multi-view oblique UAV-images, but the larger surrounding area is covered by airborne high resolution multiview images.

Although a combination of these different kinds of images seems attractive, a fully automatic and rigorous geometric processing of datasets captured from different imaging platforms, at different scale and covering different perspectives of the target object, is so far sporadically treated (Shan et al., 2013; Frahm et al., 2012; Wendel et al., 2012; Agarwal et al., 2011; Gruen et al., 2005).
In the research community a common way to evaluate new platforms, data and algorithm is to prepare and share benchmarks. The success and visibility of some benchmark activities like the Middlebury test on dense image matching (http://vision.middlebury.edu/stereo), the ISPRS WGIII/4 test on urban object detection and 3D building reconstruction (Rottensteiner et al., 2013), the KITTI suite for mobile robotics and autonomous driving research (Geiger et al., 2013) or the image matching benchmark promoted by EuroSDR (Haala, 2014), show that the provision of common datasets, in combination with a scientific task, allow researchers to objectively compare their own methods with those of others and to identify common problems and open challenges.

\subsection{Objectives of the proposed benchmark}

Inspired by these concepts, within an ISPRS Scientific Initiative and in collaboration with EuroSDR, a benchmark was proposed with the aims of creating and managing a new image dataset for the research community, consisting of different typologies of images over the same (built-up) area. In particular the project consists of acquisitions and processing of three datasets (on two test areas) that will be provided to participants (together with ground truth data): airborne oblique images, covering all 4 cardinal and the nadir directions; UAV images, consisting of nadir and oblique images; convergent and redundant terrestrial images (of some selected buildings)

The aims of the Scientific Initiative (SI) are primarily two:

(1) to provide data on different areas, focusing on different terrain and building style and epochs; 
(2) to assess the accuracy and reliability of the current methods in the calibration/orientation as well as integration of those data for dense point clouds generation and feature extraction.

Since the data will be open, other research topics, like investigation into radiometry of multi-view images (BRDF) or semantic analysis is expected and appreciated as well, but in the beginning it is not part of the benchmark as such. The evaluation of the orientation results are performed using primarily check points (CKs). The point clouds delivered by participants will be compared to ground truth data (plane fitting, cross-sections, etc.).

In order to minimize problems in conjunction with multitemporal acquisitions, data were capture within a short time difference. On the other hand, some already existing data were used as ground truth (i.e. ALS).

The project has been defined in collaboration with EuroSDR and in particular with the research activity on dense image matching (Cavegn, 2014) undertaken by EuroSDR Commission II.

An open benchmark dataset with these properties does not exist yet and it is targeted to the ISPRS and the Computer Vision communities. Moreover, this project will contribute to assess the potential of this data and their benefit and integration for mapping purposes, for instance pushing their use by public administration, NMCA or other researchers in neighbouring domains.

In this paper, a detailed description of the acquired data over the test areas will be given first. The evaluation procedures and some preliminary tests performed on the selected dataset will be then shown. A final discussion about the future benchmark developments will conclude the article.

\section{BENCHMARK AREAS AND DATA ACQUISITIONS}

Two different test areas have been surveyed over the city of Dortmund (Fig. 1 and 2). These areas have been chosen according to different criteria, such as:

(i) different types of buildings;

(ii) safety for people and instruments during the acquisitions;

(iii) easy accessibility to the whole area;

(iv) technical support and availability of local surveying companies such as the company Aerometrics (http://www.aerometrics.de) for the UAV image acquisition and the Bochum University of Applied Sciences for TLS and control point surveying by GNSS and total station;

(iv) possibility to get flight permission over build-up areas (for UAV flights).

\subsection{City center (Dortmund, Germany)}

It is a dense urban area with tall modern buildings as well as historical façades (churches and public buildings) as shown in Fig. 1. The yellow rectangle defines the area acquired by the airborne oblique image system (IGI PentaCam) while the red rectangle shows the area acquired by UAV platforms and terrestrial images as well as by TLS. This area contains besides others the Dortmund town hall and public offices. Thanks to the support of the Dortmund's cadastral authorities some markers were glued (for GCPs collection and TLS scans registration) on the windows of these buildings during the data acquisitions.

The data acquired on City centre of Dortmund are:

- Oblique images: 1260 images (252 images for each camera) were acquired with PentaCam IGI (flown by AeroWest http://www.aerowest.de) on May $19^{\text {th }}, 2014$, around approximately $10-12 \mathrm{~h}$ CET. The GSD is $10 \mathrm{~cm}$ in nadir images and varies from 8 to $12 \mathrm{~cm}$ in the oblique views. The overlap is
$75 \%$ / 80\% (along / across-track directions) for the nadir images while it is $80 \% / 80 \%$ for oblique images.

- UAV: A multi-rotor DIJ S800 was used for the data acquisition on June $8^{\text {th }}, 2014$ : 1073 images were acquired with the drone over the "Rathaus (city hall)", the "Stadthaus" as well as the obelisk and the surrounding square area. The images were acquired during 4 different flights and an image GSD ranging from 1 to $3 \mathrm{~cm}$ was adopted in each flight. Both nadir and oblique images were acquired on the area. The camera mounted on the drone was a Sony Nex-7 (the same used for terrestrial acquisitions, see below).

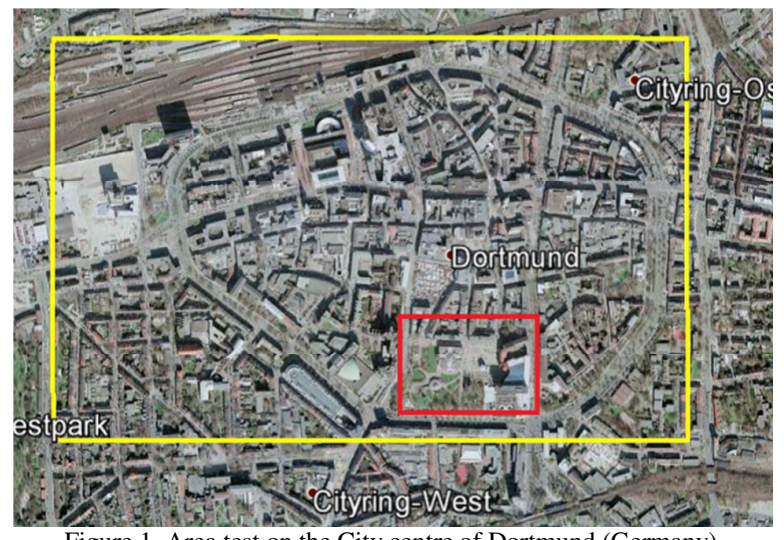

Figure 1. Area test on the City centre of Dortmund (Germany).

- Terrestrial images: Basically the same buildings as acquired with the UAV got surveyed using two different cameras on May $11^{\text {th }}$, 2014: Sony Nex-7 (2858 images) and Canon 600D with Voigtländer fix focus (1452 images). The images were acquired considering different distances from the imaged objects and using convergent and rotated views in order to enable complete objects reconstruction and to improve the reliability of the selfcalibration.

- Terrestrial Laser Scanner (TLS): The scans were acquired on May 11, 2014 using a Z+F 5010C laser scanner $(1 \mathrm{~mm}$ precision in the distance measurement). The two main buildings and the surrounding square were acquired using an average 2 $\mathrm{mm}$ resolution step on the surveyed surfaces.

- Aerial Laser Scanner (ALS): Point clouds over the test area were provided by the Municipality of Dortmund for free. The point clouds have an approximate density of $10 \mathrm{pts} / \mathrm{m}^{2}$.

- Total station (and GNSS RTK in the red area): A topographic network and a GNSS survey were set up for the scaling and geo-referencing of the imagery. These data were acquired on the same day of the terrestrial images and TLS. 17 well distributed GCPs, as well as 91 target coordinates on the building façades were surveyed. Two GNSS Leica-1200 and Leica total stations were used for this task.

\subsection{Zeche Zollern (Dortmund, Germany)}

The area (Fig. 2) was surveyed with an oblique 5-cameras system (in yellow) while a smaller part (red rectangle) was surveyed with UAV, terrestrial images as well as TLS. Targets were glued on façades of the test buildings. Most of the acquisitions (aerial, UAV, terrestrial images plus control and TLS) took place in one day: May $19^{\text {th }}, 2014$.

Data acquired on the Zeche Zollern Museum area are:

- Oblique images: 905 images taken by AeroWest with the PentaCam IGI system. The image GSDs and the overlaps are the same as listed before.

- UAV: Two different UAV platforms have been used for the image acquisition task: a DIJ S800 multi-rotor platform and a 
Mavinci fixed wing airplane. A Sony Nex-7 was installed onboard the multi-rotor system, while a (lighter) Panasonic GX1 was mounted on the Mavinci platform. The multi-rotor UAV allowed to take both nadir and oblique image views of the Zeche museum areas (1305 images) with an image GSD ranging from 1 to $3 \mathrm{~cm}$. Using the fixed-wing platform a total of 584 nadir images were captured on a wider area (i.e. the museum as well as the surrounding buildings).

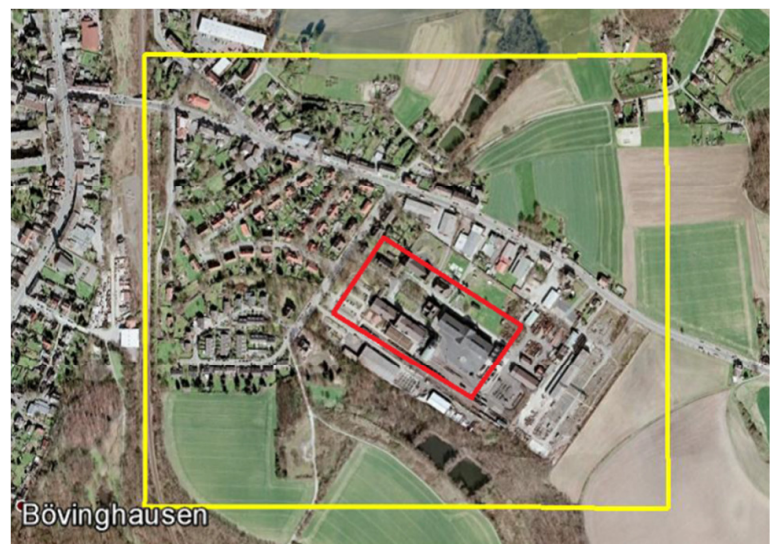

Figure 2. Area test on the Zeche Zollern Museum (Dortmund).

- Terrestrial images: Three different buildings ("Lohnhalle", "Pferdestall" and "Verwaltung") were imaged with the same cameras as above, capturing 720 images with the Canon D600 and 968 images with the Nex-7. A sub-centimeter GSD was assured in all the acquisitions.

- Terrestrial Laser Scanner (TLS): High quality scans of the objects of interest were acquired with a $\mathrm{Z}+\mathrm{F}$ 5010C laser scanner.

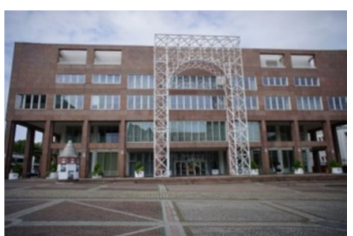

(a)

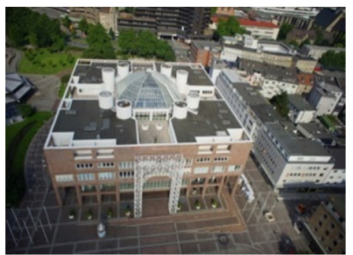

(c)

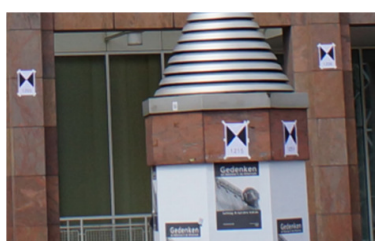

(b)

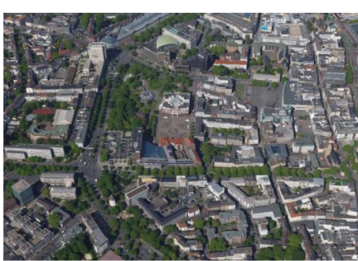

(d)
Figure 3. City centre dataset - City hall Dortmund: a) terrestrial image, b) zoom in to show some targets, c) UAV oblique image, d) IGI Pentacam image.

- Aerial Laser Scanner (ALS): Point clouds (10 pts/m2) over the area were provided by the Municipality of Dortmund.

- Total station: A topographic network was realized using Leica instruments: 176 targets on the building façades and 46 points on the ground of the museum area and the surrounding car-park were acquired as GCPs / CPs.

- GNSS acquisitions: Static acquisitions were performed on several points in the museum area to assure a better acquisition. Two Leica-1200 instruments were used for this task.

A total amount of about 2 Terabyte was collected in both areas. Fig. 3 shows some sample images from the City Centre area.

\section{DATA PRE-PROCESSING}

As the acquired datasets are very large for easy sharing, just small subsets of the available data will be initially delivered to participants. Some selected areas were manually identified in the test area.

The topographic network was refined using a Least Squares adjustment process. All the topographic points were transformed into a local Cartesian reference system.

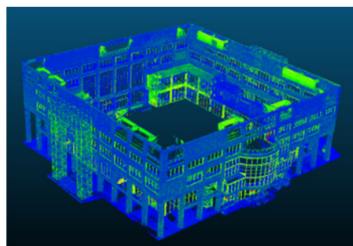

(a)

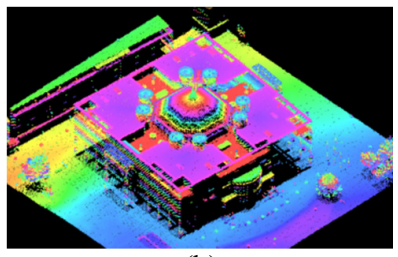

(b)
Figure 4. City hall Dortmund: a) TLS reference data (intensity color coded visualization) and b) ALS reference data (height color coded visualization).

The different TLS scans (Fig. 4 a) were co-registered using targets glued on the building façades and successively further refined by means of an ICP-based algorithm. The Z+F software was adopted for this purpose. The ALS data provided by the municipality was also registered to the same reference system (Fig. 4 b).

\section{TEST SCENARIOS AND EVALUATION CRITERIA}

In order to better synchronize this benchmark with the one described by Cavegn et al. (2014), we chose to release the dense image matching part as task $\mathrm{A}$ ), while the image orientation and calibration will follow as task B). Although in a practical setting one is normally confronted first with the image orientation problem, we see both tasks rather independently, especially in such a benchmark setup.

Six different scenarios have been set up to exploit the data acquired on both test areas and to deliver to participants reliable and challenging datasets. These tasks are described in the following sections.

Task A) Dense image matching (DIM) evaluation. This task is performed in cooperation with the EuroSDR's Scientific Initiative "Benchmark on High Density Image Matching for DSM Computation” (http://www.ifp.unistuttgart.de/EuroSDR/ImageMatching), joining both the available datasets. The efforts of both the initiative are contributing to set up a more complete and challenging dataset considering flights with different features (in terms of GSD size and overlap).

Participants' results will be evaluated according to the methodology described by Cavegn et al. (2014). The necessary images have been already oriented for the data delivery, however, no GCP or CP will be provided (those are hold back, because they are partly necessary for Task B and a publication of GCP would make the orientation assessment obsolete).

Two different scenarios are considered for the dense image matching task:

Scenario A) DIM in oblique and nadir airborne imagery: common challenges in DIM across nadir and oblique airborne imagery are related to the handling of large scale differences and occlusions. Both problems are more obvious compared to DIM in nadir image blocks alone. Moreover many algorithms initially match only in stereo and then fuse the results in object 
space. Here different approaches on how to actually do this fusion accurately, also taking into account the scale differences, are pursued.

Both datasets - the one reported in Cavegn et al. (2014), and the one described in this paper - are used for this benchmark. While the former consists of Leica RCD oblique camera system imagery, the latter one is from IGI (Pentacam). Another difference is in the overlap, which is around $80 / 80 \%$ in the latter dataset and 70/50\% in the former one.

A sub-dataset of the Zeche Zollern area has been selected. In particular the "Verwaltung" will be used for DIM evaluation. The 85 oblique images from this area and their exterior orientations, as well as camera calibration protocols, are delivered to participants. The orientation process resulted in RMSE values (on GCPs and CPs) lower than the GSD size and the bundle block adjustment report (by Pix4D (www.pix4d.com) is provided as well. The result evaluation will be performed using the TLS data of the administration building. For details on the evaluation method refer to section 5 and to (Cavegn et al., 2014).

Scenario B) DIM in a combined terrestrial and UAV image block: very detailed 3D point clouds are expected from the combination of terrestrial and UAV image blocks, showing a GSD of $2 \mathrm{~cm}$ maximum. Challenges mentioned above for scenario A hold also here, especially the fact that not only nadir and oblique, but ground-based horizontally oriented images are included, making the task even more difficult.

The UAV and terrestrial images as well as their orientations focusing on the administration building will be delivered. The image orientation and calibration has been performed with Pix4D as well. 37 control points, well distributed around and glued on the administration building itself, were used. Residual RMSE at control points are (X:5mm, Y:6mm, Z:8mm) while the residuals at independent check points (CKs) are (X:5mm, $\mathrm{Y}: 7 \mathrm{~mm}, \mathrm{Z}: 2 \mathrm{~mm})$.

In order to challenge the participants of the dense matching benchmark, it was decided not to use the complete block of 867 UAV and terrestrial images in total. Although the large number would be possibly challenging already for some methods, the number of images was reduced in order to have less redundant images in the scene, making more evident the matching errors (gaps, outliers, especially at problematic areas like sharp edges). The method proposed by Alsadik et al. (2014) was used to reduce the number of images according to coverage: given the dense point cloud processed by the Pix $4 \mathrm{~d}$ software as input, those images necessary to cover each point with 3 images were selected using this algorithm. Effectively, this means that each point is visible in at least 3 images. There might be areas where a larger coverage is realised, but the initial large redundancy is no more there.

The point clouds computed by participants will be compared to the ground truth according to the methodology referred to in Scenario A.

Task B) Image orientation evaluation. This task focuses on the image orientation assessment of different image block configurations. Both areas in Dortmund are considered, but different input data are delivered according to the goal of each conceived test. For this reason, different scenarios have been set up. For each scenario a set of images will be delivered together with GCPs coordinates. Image coordinates of both GCPs and CPs will be provided, too. Participants will be always requested to deliver, (a) average reprojection error per image, (b) CPs coordinates in object space and (c) the sparse tie-points point cloud retrieved with a bundle adjustment method. The evaluation will be performed considering the residuals on CPs and the mean deviation of sparse point cloud from reference data (TLS and ALS) on some selected planar surfaces. All scenarios are still not available to participants (February 2015), due to the lack of a complete GNSS survey that will be finished in Spring 2015.

Scenario A) Orientation of large airborne multi-view image image blocks: the whole oblique/nadir image block (1204 images, IGI Pentacam) will be provided to participants together with a set of GCPs (both image and ground coordinates) and CPs (only image coordinates). The approximate orientation achieved by direct sensor orientation solution ${ }^{1}$, and the lab camera calibration will be disclosed, too. The results evaluation will be performed on (a), (b) and (c). The sparse point cloud will be compared to some reference planes, derived from ALS and TLS (in correspondence of roof faces and large building facades) to assess the block deformations, especially in the border of the block. Details on the evaluation method will be given below in section 5 .

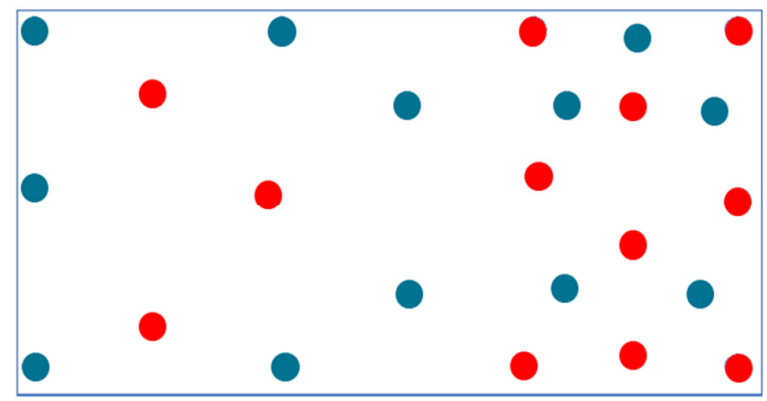

Figure 5. A schematic representation of GCPs (red dots) and CPs (blue dots) distribution. The area with well distributed GCPs is on right side of the block.

In order to better evaluate possible remaining systematic errors in the bundle solution we will NOT provide the participants with well distributed GCPs. The whole area will be divided in two halves (see Fig. 5): several well distributed GCPs will be provided in the first part to allow a reliable self-calibration of the multi-camera system and to determine CPs residuals in standard operative conditions. A small number of GCPs will be given in the second part to highlight the block deformations from the CPs residuals (Rupnik et al., 2015).

Scenario B) Orientation of large airborne multi-view image blocks, including UAV images: Scenario A will be extended adding the UAV image block to the oblique dataset. By this, the number of images within the block will increase, but the main motivation to set up a dataset with combined airborne nadir/oblique and UAV images is to test the capability of tie point matchers and bundle adjustment to cope with very large scale differences.

Only the images captured on the "Stadthaus", obelisk and the square will be considered in this dataset (the data from the city hall will be kept back for the next scenario). The approximate information provided by the onboard navigation devices will be disclosed. Again, CPs image coordinates will be delivered.

Scenario C) Image orientation and calibration across UAV and terrestrial platforms: this scenario will only focus on UAV and terrestrial images, not on airborne data. The images in correspondence to the "Rathaus" (city hall) building will be provided together with a minimum number of GCPs placed just

\footnotetext{
${ }^{1}$ According to Aerowest we can expect an error of the direct sensor
} orientation solution of about 10pix. 
on one side building and the approximate position given by the onboard GNSS devices. The image coordinates of GCPs and CPs will be provided to the users as well. As in the other scenarios, the output for the test evaluation will be (a), (b), (c).

Scenario D) Absolute image orientation of UAV images using airborne nadir and oblique imagery and their known EO/IO elements: the main goal (compared to scenario B) will be to assess the capability to correctly orient UAV images just using the oblique images, and not using GCP. This is a quite relevant scenario for practical applications. Although differential, 2phase receivers are nowadays available for UAV, still there is a need for an existing reference station. To use a reference network is not possible in all countries, and for rotary wing systems with a 2- or even 6- DOF gimbal the direct georeferencing is not solved at all. However, some (even outdated) airborne images with a georeference might be available and this scenario should help to understand the challenges when those existing images are used to co-register the UAV image block. Though it is likely that the absolute orientation of UAV images will not be in sub-pixel range since the airborne oblique have a 4-5 times larger resolution, it will be interesting how accurate and reliable tie information can be retrieved.

The oblique images including full internal and external orientation of the Zollern Zeche test area will be provided to participants as input (i.e. the same information as used in Task A, Scenario A). UAV and terrestrial images captured on the "Lohnhalle" and "Pferdestall" buildings will be provided too: both nadir flight (acquired with the Mavinci platform) and the oblique images (multi-rotor platform) will be considered. As in the former scenarios, the approximate onboard navigation data will be provided with images. Only the image coordinates of some CPs will be given together with the approximate GPS information. The evaluation will be performed in the same way as described in the other scenarios.

\section{EVALUATION PROCEDURES AND FIRST RESULTS}

For each task - image orientation and dense image matching dedicated evaluation methods were applied, afterwards briefly described together with some results. It's not the aim of the paper to rate a certain image orientation or dense image matching algorithm. Therefore the name of the used software won't be disclosed.

\subsection{Dense image matching evaluation (Task A)}

Some tests have been performed using the 2.5D-based approach to dense image assessment developed by Cavegn et al. (2014) and for a more detailed explanation, please refer to this paper.

The assessment results on a building façade of scenario A), i.e. the full PentaCam image set, are shown in Fig. 6.

The difference of the two DSMs - one retrieved from the TLS, the other from the dense image matching (DIM) on the oblique aerial images result - shown on the second row of Fig. 6 reveals that on some areas large gaps in the DIM result occur. Large deviations occur especially around edge regions, a typical problem of DIM methods. Large differences are visible on the two façade profiles too: most of the façade details are not correctly described.

The DIM in the combined UAV/terrestrial image block is - of course - much denser and more accurate than the result derived from the PentaCam images. Fig. 7 shows the same sequence of charts. The color scale is the same as in Fig. 6, therefore smaller differences are not well visible. However, in the profile some regions with quite some outliers are obvious, again mostly around edge areas.
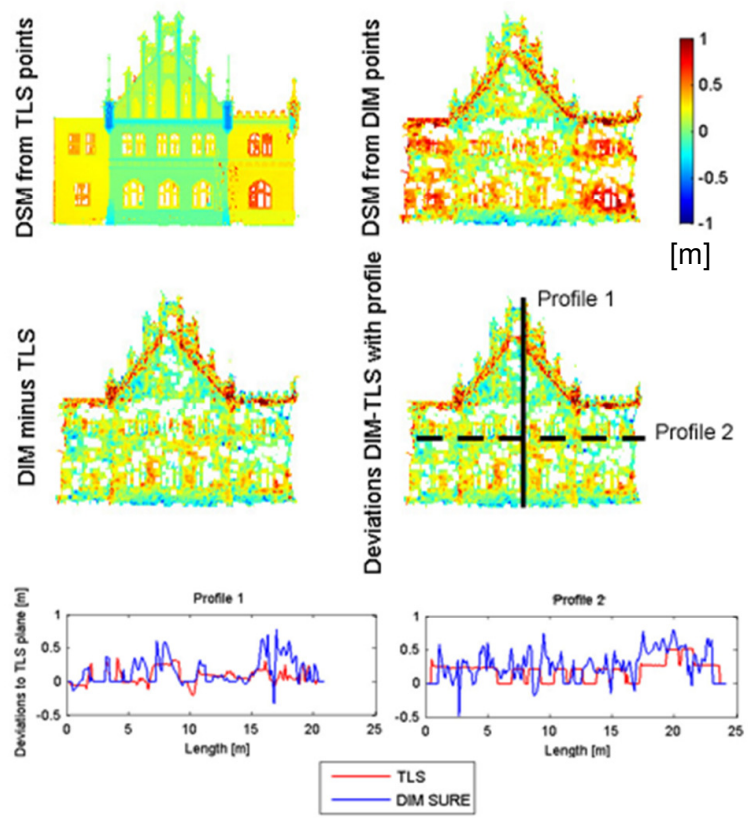

Figure 6. Example evaluation of DIM in PentaCam images.
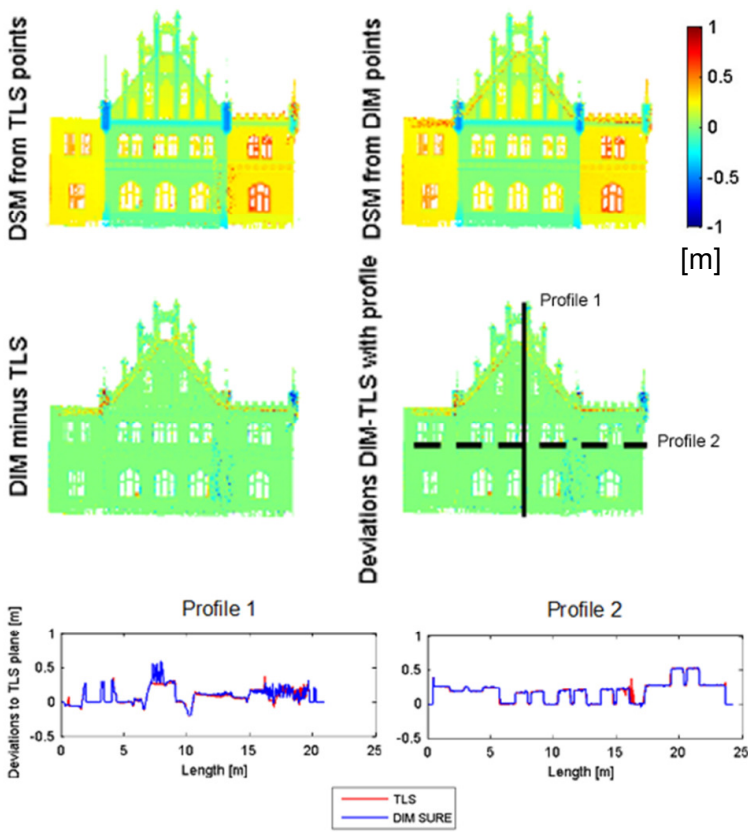

Figure 7. Example evaluation of DIM from the combined UAV/terrestrial image block.

\subsection{Image orientation evaluation (Task B)}

In order to assess the results achieved by the image orientation, every participant is able to provide the statistics values (a), (b) and (c) reported in section 4 . While the (a) and (b) values will be used in the standard way, namely to compute residuals using the reference point information, the use of (c) needs some more explanations. 
Given a reference point cloud information of a sufficient accuracy - in this case better than the expected accuracy of the tie points - we search for big planar faces within that reference. Those are for instance large roof or façade planes in TLS or ALS data.

In order to find planar faces we apply the region growing method by Vosselman (2012), then we fit planes and analyse both, the residuals and the eigenvalues. Different parameters are considered to ensure sufficiently planar faces.

First, point-to-plane residuals and their standard deviations are computed: these values must not be significantly larger than the expected mean point error. Further, we compute the curvature of the planes from the eigenvalues $L$ :

$$
C=L_{3} /\left(L_{1}+L_{2}+L_{3}\right)
$$

The curvature must be smaller than a threshold which can be derived from the ellipsoidal shape of the tensor, described by $L_{l}$ $L_{2}$ and $L_{3}$. In the sum constituting the denominator, $L_{3}$ is negligible, because from the segment growing we can already assume an approximate planar surface. In our experiments $C_{\max }=8 \mathrm{E}-5$ is used, i.e. $L_{3}$ must not be larger than $0.008 \%$ of the extent in the horizontal plane. At the same time $L_{1}$ and $L_{2}$ are requested to be larger than $3 \mathrm{~m}$.

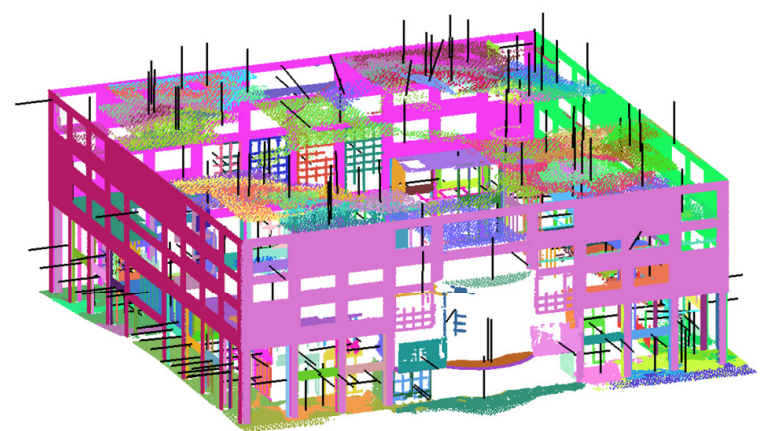

Figure 8. Selected reference planes from the "City hall" TLS/ALS data and computed plane normals. Colors refer to different planes.

For the evaluation of the actual tie points we are interested in the direction of the tie point offset to the reference plane. To do that, the normal vector direction must be defined on the reference data in order to point outside the building. This is done by computing connecting building components and assuming that the collection of planes being part of a certain component constitutes a single building entity. Hence, the normals need to point away from the centre of the respective connected component. An example of this is shown in Fig. 8: both TLS/ALS of the city hall are considered as reference in this figure. Besides the planes also the corresponding reference points are stored and used in the subsequent steps.

The individual tie points are then processed as follows: for each tie point the corresponding plane from the reference (if any) is selected. This is done by finding the closest reference point and checking if the tie point after being projected onto the plane that belongs to that reference point falls within the boundaries of the reference plane.

To do that, a rototranslation of the reference system is performed to transform the 3D problem into a $2.5 \mathrm{D}$ problem (i.e. from the model in Fig.8 to the raster map of a single façade shown in Fig. 9). This means that the local Z-coordinate of points positioned on the plane will be 0 after transformation; otherwise it will be according to the (signed) plane distance. A candidate tie point is assigned to that plane if it is surrounded by reference points in the direct vicinity. A search radius, depending on the density of reference points, is set for this test and the four closest reference points to the candidate tie point must be distributed over all four quarters (see Fig. 9). This is to ensure that a tie point does not fall into an empty area: especially at building facades where the window is excluded from the façade plane this is an important step to avoid that tie points in the window area are considered.

Only tie-points closer than a defined threshold to the reference plane are stored, while the others are discarded. The percentage of discarded tie-points in corrispondence of these surfaces is considered too.

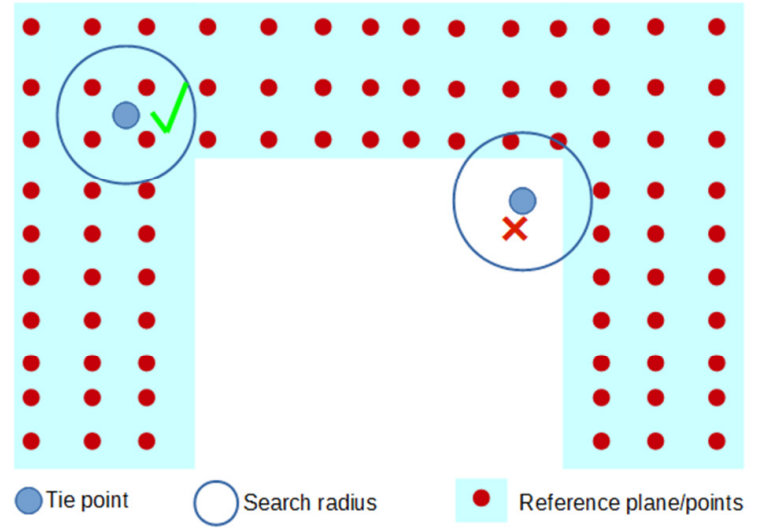

Figure 9. Filtering of tie points: only those which are surrounded by reference points are selected.

For all tie points assigned to one reference plane, the following values/statistics are computed. A schematic representation of these measures is shown in Fig. 10:

(i) the absolute and signed distance to the plane of each tie point (dN).

(ii) the mean absolute and signed orthogonal distance to tie points, including standard deviation of tie points from the corresponding reference plane.

(iii) the mean absolute horizontal and signed vertical component (dZ) of the offset (for horizonal planes only the vertical component, and for vertical planes only the horizontal offset) of tie points from the corresponding reference plane.

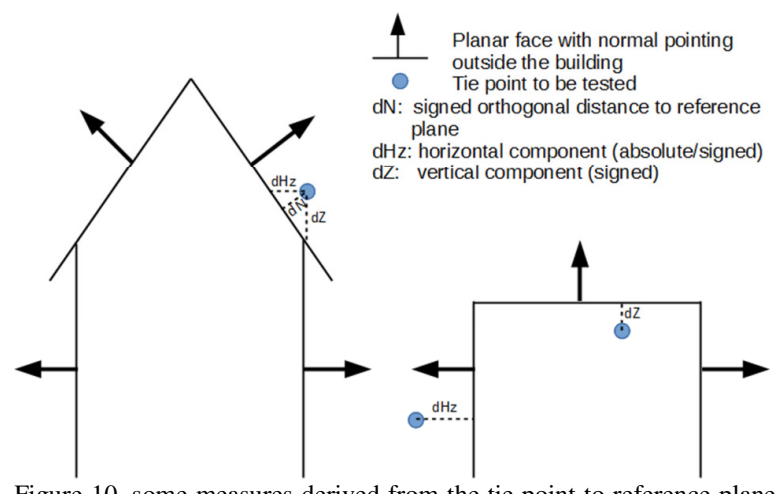

Figure 10. some measures derived from the tie point-to-reference plane assignment.

Note that by default $\mathrm{dHz}$ has no sign, because it is the horizontal projection, however depending on the sign of the mean orthogonal offset of the whole plane $\mathrm{dHz}$ is signed as well. This enables to analyse to which direction the points are shifted in case of non horizontal facades. 


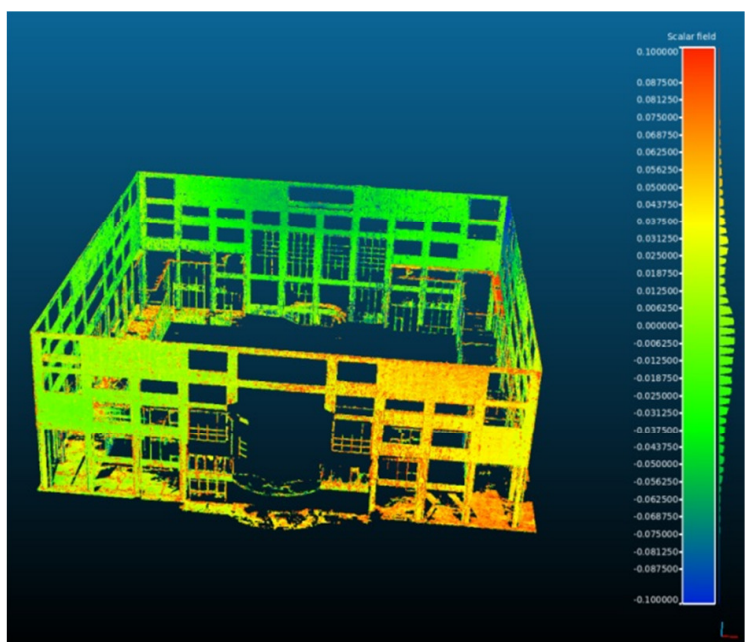

(a)

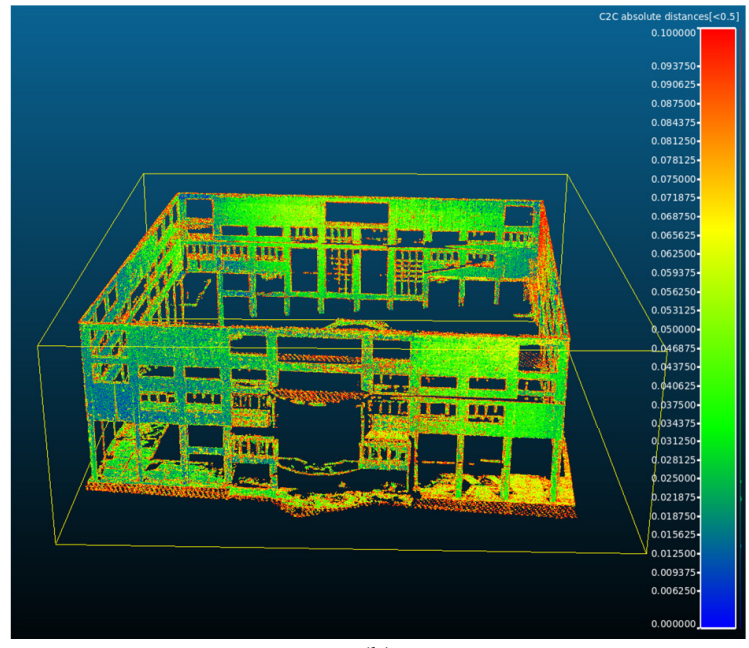

(b)

Figure 11. Evaluation of a dense point cloud: (a) signed distance from our approach, (b) absoute point-to-point distance. Maximum absolute error $10 \mathrm{~cm}$ in both cases.

In order to demonstrate the suitability of evaluation measures a dense point cloud is first considered as test data (Fig.11). The reference is the TLS dataset of the city hall. The signed distances of points to the reference planes, according to our method described in (i), are shown in Fig. 11a. On the other hand, the same dataset but just using the simple Point-to-Point asolute distance is shown in Fig. 11b.

The absolute distance value is comparable in both examples but the sign provided by the proposed method helps to further analyse the results: distances are positive on the right part of the front façade (i.e. with an offset pointing outside of the building), while the opposite happens on the other facades where distances are mostly negative, indicating some overall deformation since there the point show an inward offset.

Because of the sparse distribution of tie point clouds, the visualisation of distances from the reference data cannot be very meaningful (as shown in Fig. 12a). In these cases, the visualisation of the mean distance from each reference plane (i.e. evaluation statistics (ii) and (iii)) might be more helpful than the computation of single points indipendently.

Fig. $12 \mathrm{~b}$ shows the signed vertical distances per reference plane: point color points out the mean tie-point distances from the corresponding reference plane. A clear trend of rotation is visible: while in the front half of the object the vertical differences are negative (down to $-12 \mathrm{~cm}$, dark dots), they are in the backside positive (up to $+8 \mathrm{~cm}$, yellow dots).

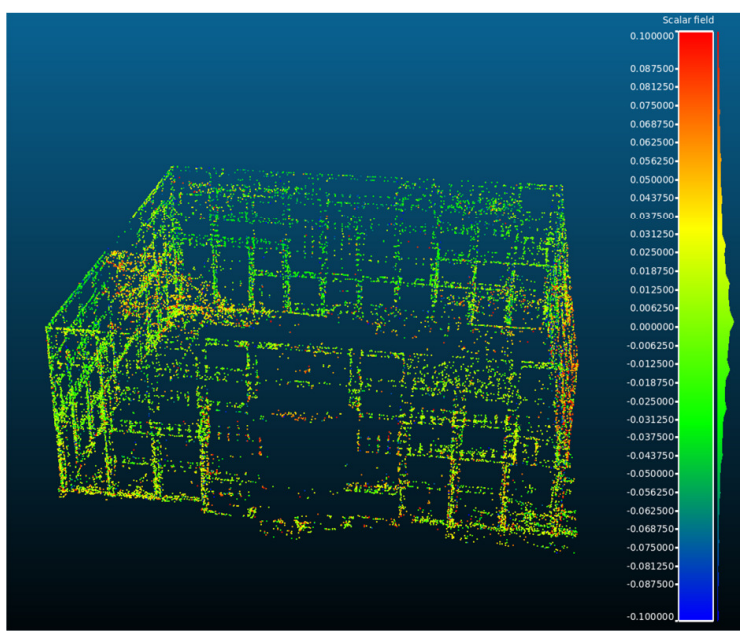

(a)

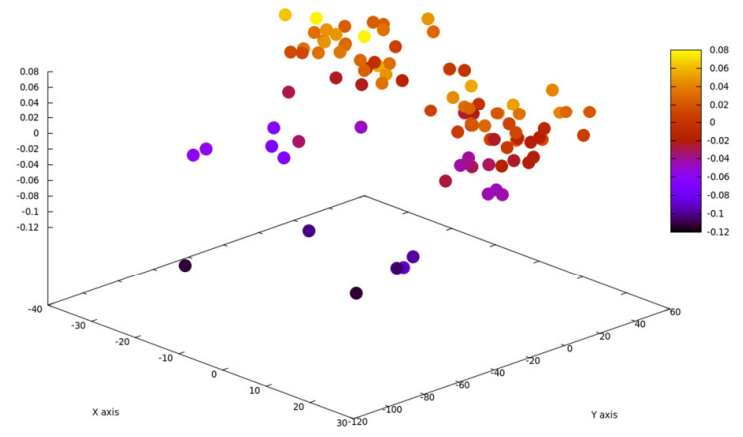

(b)

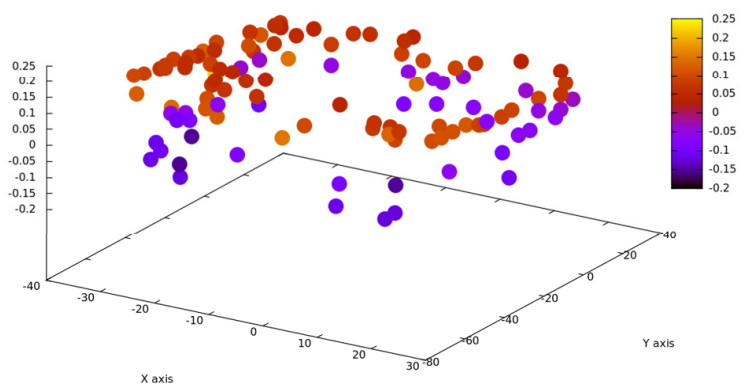

(c)

Figure 12. Evaluation of tie point quality: (a) signed orthogonal distance per each tie point, (b) signed mean vertical distance per reference plane, (c) signed mean horizontal distance per reference plane.

The horizontal offset, (Fig. 12c) indicates that in the front left area the shifts are largest (up to $20 \mathrm{~cm}$, orange dots), while in the other parts of the object they are quite small, max. $8 \mathrm{~cm}$ (violet dots).

Note that only the vertical and horizontal components are shown in Fig.12b and Fig.12c, respectively. This means that 
only the mean horizontal projection is shown for vertical façades, while only mean vertical distances are considered for flat horizontal roofs.

The developed plane-based evaluation of tie points is supposed to enable a better detection of systematic errors in the image orientation. In combination with the check points residuals this will be an efficient tool satisfying the benchmark goals.

\section{DATA DELIVERY}

A dedicated webpage has been implemented on the ISPRS website. Any participant can download the available data after a registration procedure; the procedure to upload the results is described as well. Data currently available (10 Feb. 2015) for download refer only to the image matching assessment task.

Some GNSS acquisitions in the large areas (yellow rectangles in Figs 1 and 2) still need to be captured and some data for the image orientation still need to be selected and pre-processed.

For more information, please refer to (http://www2.isprs.org/commissions/comm1/icwg15b/benchma rk_main.html) on the ISPRS's website and subscribe to the realised newsletter (http://www2.isprs.org/newsletter.html).

\section{CONCLUSIONS}

The description of a new benchmark datasets supported by ISPRS and EuroSDR and the different evaluation scenarios were presented. The proposed benchmark will deliver challenging datasets to participants in order to face some $R \& D$ open issues. The derived evaluations and conclusions will be of interest for both photogrammetric and computer vision communities. The image matching criteria has already shown to provide feasible results (Cavegn et al., 2014) in the on-going benchmark: the new airborne oblique image set (with an higher image overlap) as well as the UAV/terrestrial image block will enrich the already available dataset.

The image orientation task still needs some GNSS measurements to be completed, but it will be hopefully available before mid-2015. The evaluation criteria will be both traditional (i.e. image reprojection errors, residuals at CPs) and unconventional (i.e. tie point-to-plane distance), in order to provide a complete evaluation of the orientation results without requiring statistical measures from the bundle adjustment process. The analysis performed using these criteria have shown to be useful in all the presented case studies giving an indirect assessment of the image pose estimation quality. However, we are of course open for any discussion on the evaluation methods and assessment criteria.

The presented benchmark, in its current implementation, will be focused on image orientation and matching issues. Since the data will be open, other research topics, like investigation into radiometry of multi-view images (BRDF) or semantic analysis could be realized in the future.

\section{ACKNOWLEDGEMENTS}

Data acquisition and pre-processing was made feasible through the funds provided by ISPRS (Scientific Initiative) and EuroSDR. Further we are grateful to Aerowest $\mathrm{GmbH}$ for sponsoring part of the PentaCam flight. We thank Pix4D for providing us a research license of pix4dmapper.

\section{REFERENCES}

Agarwal, S., Furukawa, Y., Snavely, N., Simon, I., Curless, B., Seitz, S. and Szelisky, R., 2011. Building Rome in a day. In: Communication of the ACM, Vol.54(10), pp.105-112.

Alsadik, B.S., Gerke, M., Vosselman, G., Daham, A. and Jasim, L., 2014. Minimal camera networks for 3D image based modeling of cultural heritage objects. In: Sensors : journal on the science and technology of sensors and biosensors 14(4) pp. 5785-5804.

Cavegn, S., Haala, N., Nebiker, S., Rothermel, M. and Tutzauer, P., 2014. Benchmarking High Density Image Matching for Oblique Airborne Imagery. In: The International Archieves of Photogrammetry, Remote Sensing and Spatial Information Sciences, Zürich, Switzerland, Vol. XL-3, pp. 45-52.

Frahm, J., M., Georgel, P., Gallup, D., Johnson T., Raguram, R., Wu, C., Jen, Y., H., Dunn, E., Clipp, B., Lazebnik, S. and Pollefeys, M., 2010. Building Rome on a Cloudless Day. In: Computer Vision - ECCV 2010, pp. 368-381.

Furukawa, Y., Curless, B., Seitz, S.M. and Szeliski, R., 2010. Towards internet-scale multi-view stereo. In: Computer Vision and Pattern Recognition (CVPR), pp. 1434 - 1441.

Geiger, A., Lenz, P., Stiller, C. and Urtasun, R., 2013: Vision meets Robotics: The KITTI Dataset. International Journal of Robotics Research, 0(0) 1-7, DOI: 10.1177/0278364913491297

Gruen, A., Remondino, F. and Zhang, L., 2005. The Bamiyan project: multi-resolution image-based modelling. In: Recording, Modeling and Visualization of Cultural Heritage, Taylor \& Francis / Balkema, ISBN 041539208 Xpp, pp. 45-54.

Haala, N., 2014. Dense Image Matching Final Report. EuroSDR Publication Series, Official Publication No. 64, pp. 115-145.

Rottensteiner, F., Sohn, G., Gerke, M., Wegner, J.D., Breitkopf, U. and Jung, J., 2014: Results of the ISPRS Benchmark on Urban Object Detection and 3D Building Reconstruction. ISPRS Journal of Photogrammetry and Remote Sensing, 93, pp. 256271.

Rupnik, E., Nex, F., Toschi, I. and Remondino, F., 2015. Aerial multi-camera systems: Accuracy and block triangulation issues. ISPRS Journal of Photogrammetry and Remote Sensing, 101, pp. 233-246.

Shan, Q., Adams, R., Curless, B., Furukawa, Y. and Seitz, S. M., 2013. The Visual Turing Test for Scene Reconstruction. Proceedings of: 3DV '13, pp. 25-32.

Vosselman, G., 2012. Automated planimetric quality control in high accuracy airborne laser scanning surveys. ISPRS Journal of Photogrammetry and Remote Sensing, 74, pp. 90-100.

Wendel, A., Hoppe, C., Bischof, H. and Leberl, F., 2012. Automatic fusion of partial reconstructions. In: ISPRS Annals of Photogrammetry, Remote Sensing and Spatial Information Sciences, I-3, pp.81-86. 